\title{
Model of Coupled Drives Apparatus - Static and Dynamic Characteristics
}

\author{
Petr Chalupa ${ }^{a}$, Jakub Novák and Michal Jarmar \\ Faculty of Applied Informatics, Tomas Bata University in Zlin, 76001 Zlin, Czech Republic
}

\begin{abstract}
The paper presents an initial part of a modelling the Coupled Drives Apparatus CE108 developed by TecQuipment Ltd. The final model should be used in the process of control design for the apparatus. The model derived by the developers of the apparatus using first-principle modelling is used as a starting point for comparison of its properties with measured characteristics. The static and dynamic characteristics of the real-time apparatus are presented and discussed. Special attention is paid to principal differences between the model and the real-time apparatus. Realtime measurements are obtained using MATLAB / Simulink environment in connection with a Real-time Toolbox. Evaluation of the experiments is also performed in the MATLAB environment.
\end{abstract}

\section{Introduction}

Most current control algorithms use a model of a controlled plant [1]. It is obvious that some information about controlled plant is required to allow for design of a controller with satisfactory performance. A plant model can be also used to investigate properties and behaviour of the modelled plant without a risk of damage or violating technological constraints of the real plant. There are two basic approaches of obtaining plant model: the black box approach and the first principles modelling (mathematicalphysical analysis of the plant).

The black box modelling [2], [3] is based on analysis of input and output signals of the plant. The main advantage of the black box approach consists in the possibility of usage the same identification algorithm for wide set of various controlled plants [4], [5].

On the other hand, the first principle modelling provides general models valid for whole range of plant inputs and states. A first principle model is created by analysing the modelled plant and combining physical laws [6]. Regrettably there are usually many unknown constants and relations when performing analysis of a plant. Therefore, first principle models are suitable for simple controlled plants with small number of parameters. First principle modelling can be also used for obtaining basic information about controlled plant (rage of gain, rank of suitable sample time, etc.). Some simplifications must be used to obtain reasonable results in more complicated cases. These simplifications must relate with the purpose of the model. The first principle modelling is also referred to as white box modelling.

The CE108 apparatus, which was developed by TecQuipment Ltd., is modelled by combination of both methods. Basic relations between plant inputs and outputs are taken over from the first-principle model derived by developers of the apparatus. The obtained model is confronted with the data obtained by real-time measurements. The model will be further improved to represent the real-time apparatus well. This approach is known as grey box modelling [7].

The goal of the whole work is to obtain a mathematical model of the CE108 Couple Drives Apparatus and design the model in MATLAB-Simulink environment. The resulting model should be used in the process of design of the controllers for the real-time control of the CE108 apparatus A model, which represents the plant well, can considerably reduce testing time of different control approaches because only promising control strategies are applied to the real plant and verified.

Controlling the tension and speed of interacting drive systems is a common task in industrial applications. The manufacture of continuous lengths of material can serve as an example. Textiles, paper, wire, metal sheet, plastic films are all processed in continuous lengths. The material is transported and processed through work stations by drive systems and the material speed and tension to be controlled to within defined limits at all times. In applications such as steel rolling mills the material becomes thinner and stretched as it passes through each roller. Each roller therefore has to be set at different speeds. [8]

The paper is organized as follows. Section 2 presents the modelled system - CE108 Coupled Drives Apparatus. The initial first-principle model is presented Section 3. Section 4 on focused on measurement of static and dynamic characteristics of the real-time apparatus. Finally, differences between the model and measured data are discussed in Section 5.

\footnotetext{
a Corresponding author: chalupa@fai.utb.cz
} 


\section{Coupled drives apparatus}

A photo of the CE108 Coupled Drives Apparatus is presented in Figure 1.

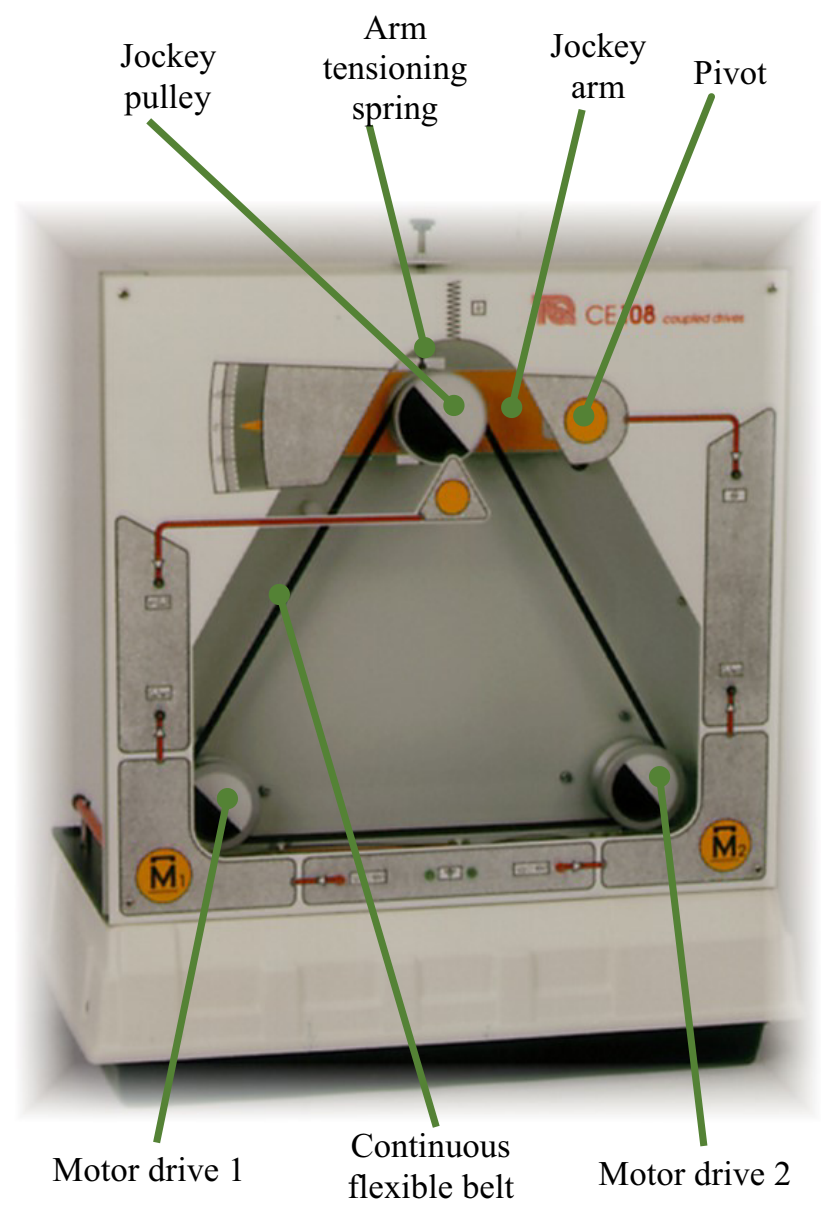

Figure 1. CE108 Coupled Drives Apparatus.

The apparatus consists of two drive motors (Motor 1 and Motor 2). These drives ae used to control the speed and the tension of a continuous flexible belt. The belt goes round three pulleys. Two of them are attached on the drive motor shafts and a jockey pulley. The jockey pulley is mounted on a swinging arm that is supported by a spring. The position of the arm corresponds to the tension in the drive belt [8].

The CE108 Coupled Drives Apparatus has two input signals:

- control voltage of motor M1,

- control voltage of motor M2.

The apparatus provides 4 output signals:

- speed of the belt, i.e. rotations of the jockey pulley,

- tension of the belt measured as an angle of the arm,

- rotations of the motor M1,

- rotations of the motor M2.

The rotations of the motors can also be considered states of the apparatus.

In the most often used setup, the apparatus is considered a Two Inputs Two Outputs (TITO) system with control voltages as the inputs and belt speed and tension as the outputs.

\section{Initial first principle model}

A scheme of the CE108 Coupled Drives Apparatus is shown in Figure 2 [8].

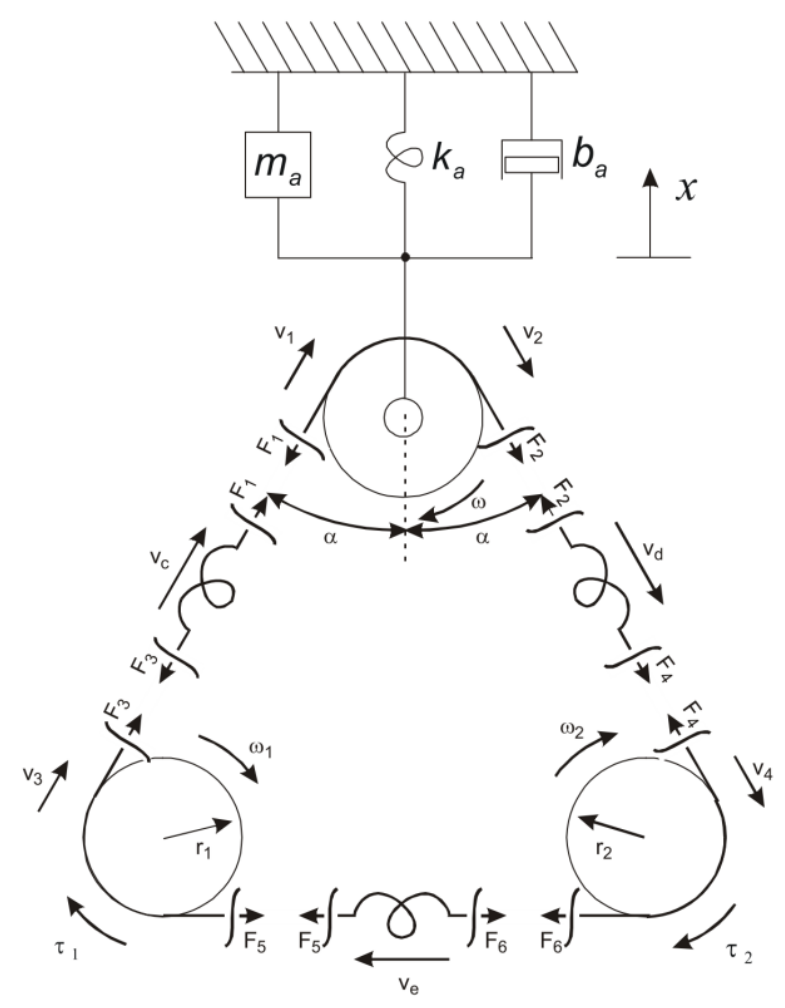

Figure 2. Scheme of the CE108 Coupled Drives Apparatus.

\subsection{Notation}

The following notation is used for derivation of the model of the apparatus:

$m_{\mathrm{a}}$ - the jockey arm and pulley mass,

$k_{a}$ - the jockey arm spring stiffness,

$b_{a}$ - the jockey arm bearing coefficient of friction,

$x$ - the vertical position of the jockey pulley,

$\alpha$ - the angle of the belt sections with the vertical,

$F_{a}$ - vertical force affecting the spring,

$k$ - the stiffness of the belt,

$I$ - the moment of inertia of a motor drive,

$b$ - the friction coefficient of inertial of a motor drive,

$\omega_{i}$ - the rotational speed (angular velocity) of Drive $i$; $i \in\{1,2\}$,

$\omega$ - the rotational speed (angular velocity) of the jockey pulley,

$\tau_{i}$ - the input torque of Drive $i ; i \in\{1,2\}$,

$F_{i}$ - the tensions in the individual belt sections; $i \in\{1,2$, $3,4,5,6\}$,

$x_{c}$ - the extension of the belt section $\mathrm{C}$,

$x_{d}$ - the extension of the belt section $\mathrm{D}$,

$x_{e}$ - the extension of the belt section $\mathrm{E}$,

$v_{i}$ - velocity of the belt at a corresponding position; $i \in\{1$, $2,3,4\}$, 
$p$ - the momentum of mass $m_{a}$,

$h_{i}-$ the drive pulley/motor angular momenta; $i \in\{1,2\}$.

\subsection{Derivation of the model}

This section presents short form of derivation of a first principle model of the CE108 apparatus. Details can be found in [8] and [9].

It is assumed that the pulley is light and rotates without friction:

$$
F_{1}=F_{2}=F
$$

Then vertical force affecting the spring can be written as follows:

$$
F_{a}=2 F \cos (\alpha)
$$

And from the conservation of power:

$$
F_{a} \dot{x}=F\left(v_{1}-v_{2}\right)
$$

Combining (2) and (3) leads to

$$
2 \dot{x} \cos (\alpha)=v_{1}-v_{2}
$$

The vertical force balance on jockey arm assembly leads to:

$$
F_{a}=\dot{p}+k_{a} x+b_{a} \dot{x}
$$

The force balance on belt sections $\mathrm{C}$ and $\mathrm{D}$ gives

$$
F=k x_{c}=k x_{d}
$$

where

$$
F=F_{1}=F_{2}=F_{3}=F_{4}
$$

The force balance of the belt in section $\mathrm{E}$ is described by (8):

$$
F^{\prime}=k x_{e}
$$

where

$$
F^{\prime}=F_{5}=F_{6}
$$

A torque balance on the drive pulleys gives

$$
\begin{aligned}
& \tau_{1}+F_{3} r-F_{5} r=\dot{h_{1}}+b \omega_{1} \\
& \tau_{2}+F_{6} r-F_{4} r=\dot{h_{2}}+b \omega_{2}
\end{aligned}
$$

where

$$
\begin{aligned}
& h_{1}=I \omega_{1} \\
& h_{2}=I \omega_{2}
\end{aligned}
$$

Angular velocities of motors and belt velocities are connected according to the following equations:

$$
\begin{aligned}
& v_{3}=\omega_{1} r \\
& v_{4}=\omega_{2} r
\end{aligned}
$$

Combining equations (4) - (15) leads to state space description of the CE108 Coupled Drives Apparatus.

$$
\begin{aligned}
\dot{h}_{1} & =-\frac{b}{I} h_{1}+r k x_{c}-r k x_{e}+\tau_{1} \\
\dot{h}_{2} & =-\frac{b}{I} h_{2}-r k x_{c}+r k x_{e}+\tau_{2} \\
\dot{x}_{c} & =\frac{1}{2}\left(-h_{1} \frac{r}{I}+h_{2} \frac{r}{I}+p \frac{2 \cos (\alpha)}{m_{a}}\right) \\
\dot{x}_{e} & =\frac{r}{I} h_{1}-\frac{r}{I} h_{2} \\
\dot{x} & =\frac{p}{m_{a}} \\
\dot{p} & =2 \cos (\alpha) k x_{c}-k_{a} x-\frac{b_{a}}{m_{a}} p
\end{aligned}
$$

It is possible to derive two transfer functions from the state equations:

$$
\begin{gathered}
\omega(s)=\frac{\tau_{1}(s)+\tau_{2}(s)}{2(s I+b)} \\
x(s)=\frac{k r \cos (\alpha)\left(\tau_{2}(s)-\tau_{1}(s)\right)}{\left(I s^{2}+b+3 k r^{2}\right)\left(m_{a} s^{2}+b_{a} s+k_{a}+2 k \cos ^{2}(\alpha)\right)-2 k^{2} r^{2} \cos ^{2}(\alpha)}(18)
\end{gathered}
$$

The motor torques $\tau_{1}$ and $\tau_{2}$ are derived by the input signals $u_{1}$ and $u_{2}$. In the simplest case the motor actuator characteristic is linear:

$$
\begin{aligned}
\tau_{1} & =g_{1} u_{1} \\
\tau_{2} & =g_{2} u_{2}
\end{aligned}
$$

The jockey arm deflection and belt speed are measured by a sensor with outputs $y_{x}$ and $y_{\omega}$ respectively. The characteristics of the sensors is assumed to be linear:

$$
\begin{gathered}
y_{x}=g_{x} x \\
y_{\omega}=g_{\omega} \omega
\end{gathered}
$$

It should be noted that it is assumed that the friction coefficients between the belt and motor pulleys is the same for both motor pulleys.

Combining equations (17) - (22) leads to the following statements concerning static characteristics of the system:

- The speed of the belt in the steady states is proportional to the sum of control inputs $\left(u_{1}+u_{2}\right)$.

- The tension of the belt in the steady states is proportional to the difference between control inputs $\left(u_{2}-u_{1}\right)$

- The system is linear with respect to its control inputs

\section{Static and dynamic characteristics}

This sections presents several measurements of static and dynamic characteristics of the CE108 Coupled Drives Apparatus.

The measurements were performed using MATLAB / Simulink environment. The real-time behaviour of Simulink was guaranteed by using Real-Time Toolbox by Humusoft Ltd. A data acquisition PCI card MF624 was used as an interface between personal computer and the apparatus.

The measurements of static characteristics were performed for both increasing and decreasing sequence of 
control signal. Fast sampling time of $T_{0}=0.01 \mathrm{~s}$ was used for all measurements. Static characteristics was measured more than 30 times to obtain representative results for different setups. Typically, each setup was measured 3 times and the results were compared to state if averaging can be used.

An example of input signals courses is presented in Figure 3.

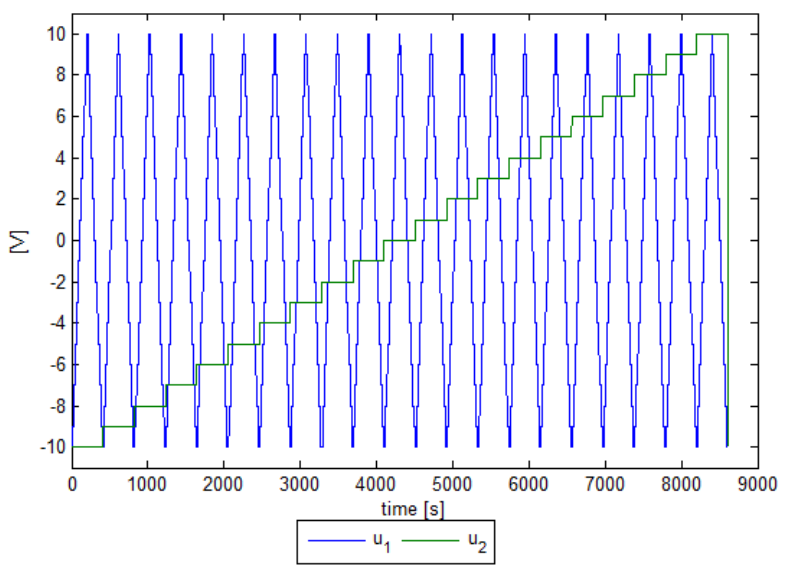

Figure 3. Course of input signals for measurement of static characteristics.

It can be seen that both signals are piece-wise constant. The signal $u_{2}$ is constant for the whole increase-decrease cycle of the signal $u_{1}$. Hence, the input $u_{1}$ is stated as the one with faster changes of its value. This single measurement lasted for 8610 s (i.e. almost 2,5 hours).

A typical static characteristics of belt speed is presented in Figure 4. In this case control inputs were coursed according to Figure 3. Steady states for increasing sequence of $u_{1}$ is presented.

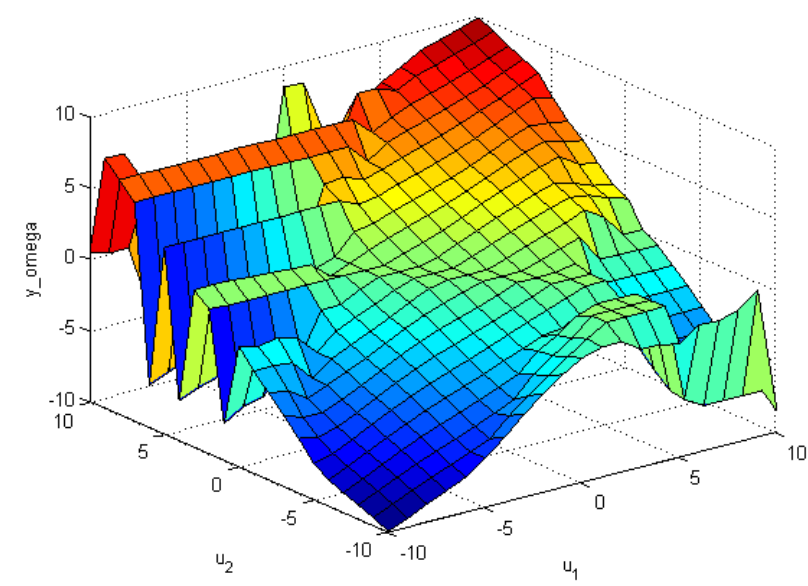

Figure 4. Static characteristics of belt speed (increasing $u_{1}$ )

It can be observed that the system is not linear especially in the area where signs of input signals is different (i.e. $u_{1}<0$ and simultaneously $u_{2}>0$ or $u_{1}>0$ and simultaneously $u_{2}<0$ ). A linear area is observed in the neighbourhood of line $u_{1}=u_{2}$ with a small dead-zone close to origin $\left(u_{1}=0, u_{2}=0\right)$.

The static characteristics for decreasing $u_{1}$ is presented in Figure 5.

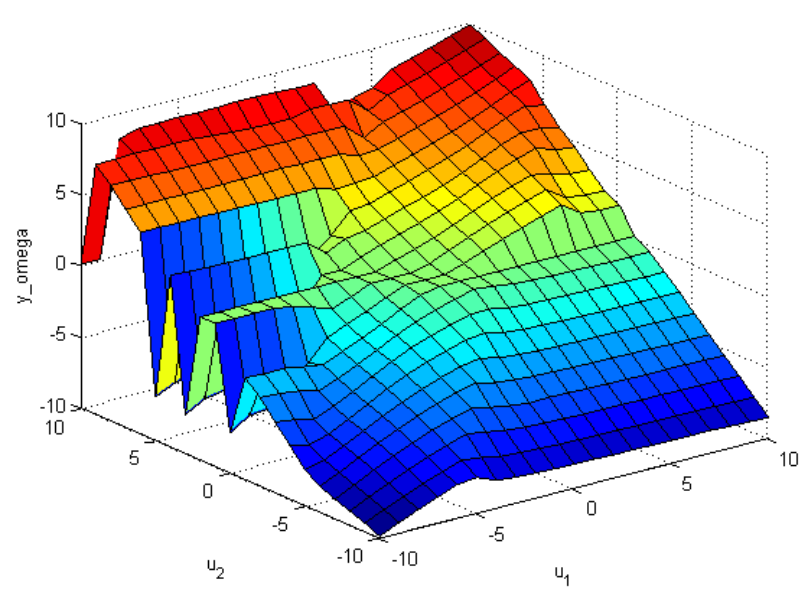

Figure 5. Static characteristics of belt speed (decreasing $u_{1}$ )

It can be observed from comparison of Figure 4 and Figure 5 that the output value of the steady CE108 apparatus is dependant not only on the current steady inputs but also on their history. This statement is supported by measurement of static characteristics where input $u_{2}$ was changing faster - the courses of inputs were opposite to Figure 3. Resulting static characteristics of belt speed is presented in Figure 6.

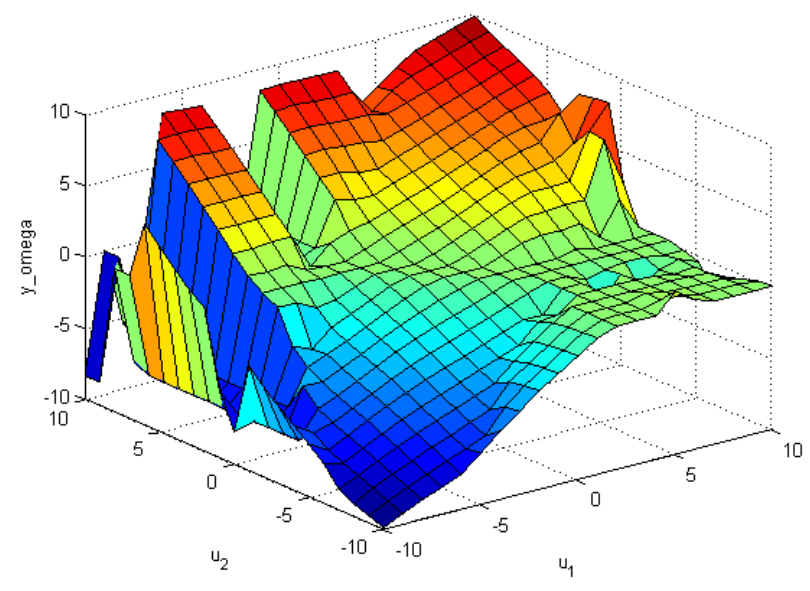

Figure 6. Static characteristics of belt speed (increasing $u_{2}$ )

As the system behaviour was studied deeper it was found out that that for particular combinations of steady inputs the belt speed tends to be close to the speed of one of motors regardless of the speed of the other motor.

This situation can be observed in Figure 7. It can be seen that the bet speed is:

- close to speed of motor M1, or

- close to speed of motor M2, or

- close to zero.

Belt speed proportional to sum of input signal did not occur in this part of the measurement of static characteristics.

The same course with smaller time frame is presented in Figure 8. This figure can be used to study dynamic behaviour of the apparatus as the course contains several step responses of both belt speed and belt tension. 


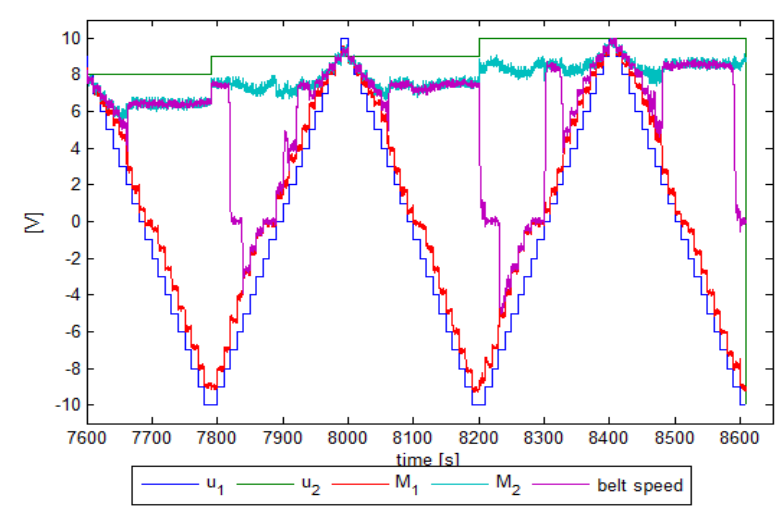

Figure 7. Measurement of static characteristic - course of input and output signals

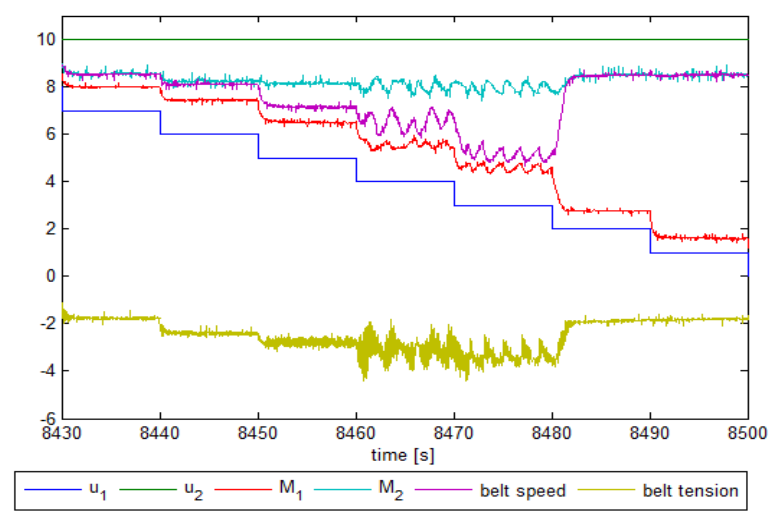

Figure 8. Dynamic behaviour of the CE108 apparatus

A typical static characteristic of belt tension is presented in Figure 9.

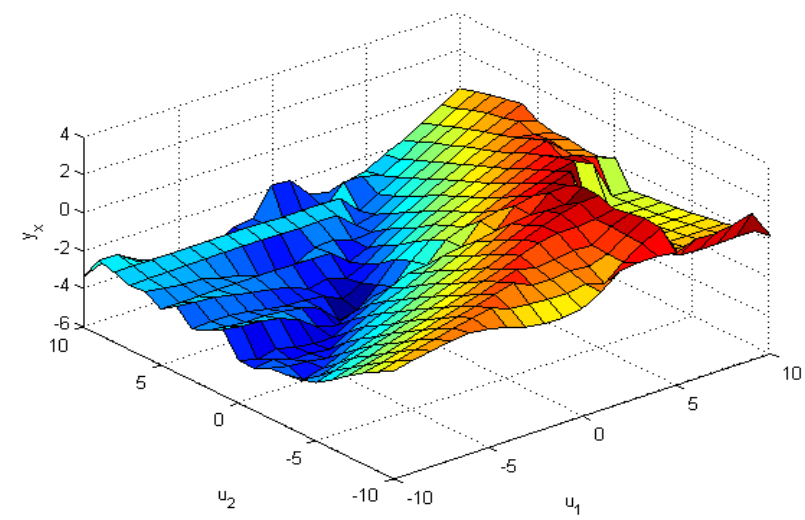

Figure 9. Typical static characteristics of belt tension

As for the linearity of the belt tension, the statement might be the same as for belt speed: A linear area is observed in the neighbourhood of line $u_{1}=u_{2}$.

\section{Comparison of model and real-time apparatus}

Several principal differences were observed when comparing behaviour of the first principle model with realtime measurements:
- The CE108 apparatus is not linear in whole range of its inputs

- The steady outputs of the CE108 apparatus do not depend only on the steady inputs but also on the previous course of the inputs

- The behaviour of the CE108 apparatus in the area where inputs have opposite sign is very far from deterministic. This area can be modelled only by stochastic approach

- Steady outputs are not reached for the steady inputs in some cases. This behaviour represents nonlinearity of the system (see Figure 8).

\section{Conclusion}

The CE108 Coupled Drives Apparatus was presented as well as its first principle model. Many static characteristics of the real-tome apparatus were measured and the behaviour of the real-time system was compared with the model. The comparison was focused on principal differences which cannot be omitted or suppressed by tuning parameters of the model. Significant differences occur especially in cases where the signs of the input were opposite.

Further work will be focused on improvement of the model to represent nonlinearities of the CE108 Coupled Drives Apparatus.

\section{Acknowledgment}

This work was supported by the Ministry of Education, Youth and Sports of the Czech Republic within the National Sustainability Programme project No. LO1303 (MSMT-7778/2014).

\section{References}

1. V. Bobál, J. Böhm, J. Fessl, J. Macháček, Digital Selftuning Controllers: Algorithms, Implementation and Applications (2005)

2. G. P. Liu, Nonlinear identification and control - A neural network Approach (2001)

3. L. Ljung, System identification: theory for the user. Upper Saddle River (1999)

4. J. Mikleš, M. Fikar, Process modelling, identification, and control (2007)

5. B. Codrons, Process modelling for control: a unified framework using standard black-box techniques (2005)

6. D. M. Himmelblau. J. B. Riggs, Basic principles and calculations in chemical engineering (2004).

7. K.C. Tan, Y. Li, Control Engineering Practice 10, 673-684 (2002).

8. H. Hagadoorn, M. Readman, Coupled Drives 1: Basics (2004), http://www.control-systemsprinciples.co.uk/whitepapers/coupled-drives1.pdf

9. H. Hagadoorn, M. Readman, Coupled Drives 2: Control and analysis (2004), http://www.controlsystems-principles.co.uk/whitepapers/coupleddrives2.pdf 